\title{
Supporting Information: Asymmetric Bubble Formation at Rectangular Orifices
}

\author{
Yujia Zhou ${ }^{1,2}$, Bingqiang $\mathrm{Ji}^{1, *}$, Xiao Yan ${ }^{1}$, Puhang $\mathrm{Jin}^{1}$, Jiaqi $\mathrm{Li}^{1}$, \\ Nenad Miljkovic ${ }^{1,3,4,5, *}$ \\ ${ }^{1}$ Mechanical Science and Engineering, University of Illinois at Urbana Champaign, Urbana, \\ Illinois 61801, USA \\ ${ }^{2}$ Current Address: Institute of Nuclear and New Energy Technology, Advanced Nuclear Energy \\ Technology Cooperation Innovation Centre, Key Laboratory of Advanced Nuclear Engineering \\ and Safety, Tsinghua University, Beijing 100084, China \\ ${ }^{3}$ International Institute for Carbon Neutral Energy Research (WPI-I2CNER), Kyushu University, \\ 744 Moto-oka, Nishi-ku, Fukuoka 819-0395, Japan \\ ${ }^{4}$ Electrical and Computer Engineering, University of Illinois at Urbana Champaign, Urbana, \\ Illinois 61801, USA \\ ${ }^{5}$ Materials Research Laboratory, University of Illinois at Urbana Champaign, Urbana, Illinois \\ 61801, USA \\ * Corresponding authors. Email: jbq@illinois.edu and nmiljkov@illinois.edu
}

\section{Contents}

Derivation of the bubble detachment size formula in dynamic regimes

\section{References}




\section{Derivation of the bubble detachment size formula in dynamic regimes}

The part of the bubble above the neck was taken as the object of interest of the force balance model. Following the work of Ma et al. ${ }^{\mathrm{S} 1}$, in the dynamic regime, the bubble experiences the buoyant force $F_{B}$, surface tension force $F_{S}$, (gas) inertia force $F_{I}$, virtual mass force $F_{V}$, and drag force $F_{D}$, where

$$
\begin{gathered}
F_{B}=\left(\rho_{l}-\rho_{g}\right) V g, \\
F_{S}=-2 \sigma\left(w_{n}+l_{n}\right), \\
F_{I}=-\frac{d\left(\rho_{g} V u_{b}\right)}{d t}=-\rho_{g} V \frac{d u_{b}}{d t}-\rho_{g} u_{b} u A_{n}, \\
F_{V}=-C_{V M} \rho_{l} V \frac{d u_{b}}{d t} .
\end{gathered}
$$

Here, $t$ is time, $u_{b}$ is the bubble velocity, $C_{V M}=0.5$ is the virtual mass coefficient, $A_{n}=\ln _{n} w_{n}$ is the cross-section area of the bubble neck, $u$ is the gas velocity at the neck section calculated by $u=$ $Q /\left(l_{n} w_{n}\right)$, and $V=u A_{n} t$ is the bubble volume. We estimate the bubble velocity $u_{b}$ with the bubble top velocity, which was found to be $\leq O\left(10^{-2} \mathrm{~m} / \mathrm{s}\right)$, yielding a Reynolds number of $R e=\rho l u b d / \mu l \leq$ $O(10)$. Thus, we estimate the drag force as:

$$
F_{D} \sim d \mu_{l} u_{b} .
$$

Because $\rho_{g} u_{b} u A_{n} / F_{S}=\rho_{g} u b u l_{n} w_{n} /\left(2 \sigma\left(l_{n}+w_{n}\right)\right)<O\left(10^{-3}\right)$, the second term of the inertia force $F_{I}$ can be safely neglected compared to the surface tension force $F_{s}$. Meanwhile, many past works have found that the bubble detachment size in an inviscid liquid is independent of or very weakly affected by the liquid viscosity $\mu l$. The past works have suggested that the viscous drag $F_{D}$ can be neglected $^{\mathrm{S} 2, \mathrm{~S} 3, \mathrm{~S} 4}$. This argument can be easily understood if we compare $F_{D}$ with $F_{S}$ by the resulting capillary number $C a=\mu l u_{b} d /\left(\sigma\left(l_{n}+w_{n}\right)\right)<O\left(10^{-3}\right)$, which means viscous forces are 
negligible compared to $F_{S}$. Therefore, the force balance equation can be expressed as

$$
F_{B}+F_{S}+F_{V}+F_{I}=0
$$

which can be further expressed as

$$
\frac{d u_{b}}{d t}=\frac{\left(\rho_{l}-\rho_{g}\right) g}{C_{V M} \rho_{l}+\rho_{g}}-\frac{2 \sigma\left(w_{n}+l_{n}\right)}{C_{V M} \rho_{l}+\rho_{g}} \frac{1}{V} .
$$

Previous studies have suggested that the expansion stage of the dynamic regime can be divided into two stages $^{\mathrm{S} 2, \mathrm{~S} 5}$, as shown in Figure S1. In the first stage, the bubble grows on the orifice with a bubble moving velocity of $u_{b}=0$ (negligible dynamic forces) until the beginning of the second stage when the buoyancy $F_{B}$ and surface tension $F_{S}$ balance each other, i.e.:

$$
\rho_{l} V_{s} g=2 \sigma\left(l_{n}+w_{n}\right),
$$

where $V_{s}=\pi d_{s}^{3} / 6$ is the quasi-static bubble detachment volume. We define the time duration of the first stage as $t_{0}$, and then $V_{s}=u A_{n} t_{0}$. By introducing the dimensionless parameters of $u^{*}=u b / u$, $V^{*}=V / V_{s}$, and $t^{*}=t / t_{0}$, Eq. (S7) can be expressed as

$$
\frac{d u^{*}}{d t^{*}}=\frac{\left(\rho_{l}-\rho_{g}\right) g V_{s}}{\left(C_{V M} \rho_{l}+\rho_{g}\right) u^{2} A_{n}}-\frac{2 \sigma\left(w_{n}+l_{n}\right)}{\left(C_{V M} \rho_{l}+\rho_{g}\right) u^{2} A_{n}} \frac{1}{V^{*}} .
$$

Considering $\rho_{l} \gg \rho_{g}$ and $w=w_{n}$ and substituting Eq. (S8) into Eq. (S9), we have

$$
\frac{d u^{*}}{d V^{*}}=\frac{d u^{*}}{d t^{*}}=\frac{1}{W e}-\frac{1}{W e} \frac{1}{V^{*}},
$$

with the Weber number

$$
W e=\frac{C_{V M} \rho_{l} u^{2} l_{n} w}{2 \sigma\left(l_{n}+w\right)} .
$$

The integral of Eq. (S10) at the second stage of the expansion stage can be written as:

$$
\int_{0}^{1} d u^{*}=\int_{1}^{V_{d}^{*}} \frac{1}{W e} d V^{*}-\int_{1}^{V_{d}^{*}} \frac{1}{W e} \frac{1}{V^{*}} d V^{*},
$$


where $V_{d} *=V_{d} / V_{s}=\left(d_{d} / d_{s}\right)^{3}$ is the dimensionless bubble detachment volume in dynamic regimes. Finally, we obtain:

$$
V_{d}^{*}-1-\ln V_{d}^{*}=W e
$$

which is equivalent to Eq. (9) in the manuscript.

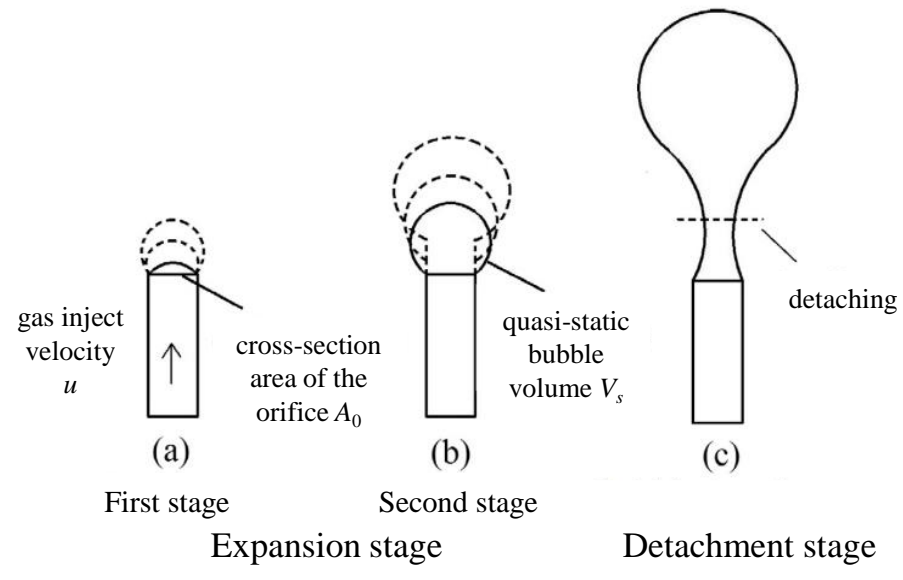

Figure S1. Sketch of the two-stage model of dynamic bubble formation at an axisymmetric orifice. The bubble formation consists of two stages of expansion $(a, b)$ and detachment (c), where the expansion stage in the dynamic regimes can be further divided into two stages shown in (a) and (b).

\section{References}

(S1) Ma, J.; Li, J.; Zhou, P.; Song, Y.; Chai, L.; Zhou, C. Q., A viewpoint on the dynamics of bubble formation from a submerged nozzle. Eur. J. Mech. B-Fluids 2019, 78, 276-283.

(S2) Kumar, R.; Kuloor, N. K., The Formation of Bubbles and Drops. Advances in Chemical Engineering, 1970, 8, 255-368.

(S3) Li, H. Z., Mouline, Y., \& Midoux, N., Modelling the bubble formation dynamics in nonNewtonian fluids. Chem. Eng. Sci. 2002, 57(3), 339-346.

(S4) Di Bari, S., Lakehal, D., \& Robinson, A. J., A numerical study of quasi-static gas injected 
bubble growth: Some aspects of gravity. Int. J. Heat Mass Tran. 2013, 64, 468-482.

(S5) Kulkarni, A. A.; Joshi, J. B., Bubble Formation and Bubble Rise Velocity in Gas-Liquid Systems: A Review. Ind. Eng. Chem. Res. 2005, 44 (16), 5873-5931. 